\title{
Atividade reprodutiva de Scinax rizibilis (Bokermann) (Anura, Hylidae) na Floresta Atlântica, sudeste do Brasil
}

\author{
Rogério P. Bastos 1 \\ Célio F. B. Haddad ${ }^{2}$
}

\begin{abstract}
Breeding activity of the treefrog Scinax rizibilis (Bokermann) (Anura, Hylidae) in the Atlantic Forest, Southeastern, Brazil. The breeding activity of Scinax rizibilis (Bokermann, 1964) was observed in a temporary pond, in the municipality of Ribeirão Branco, in the Southern São Paulo State from February 1993 to January 1994 and November 1994 to February 1995. The reproductive season extended from September to February. Females were larger and heavier (after oviposition) than males. In the chorus, the spatial distribution tended towards uniformity. The number of males and females in the chorus was positively correlated. However there was no correlation between the number of males and the estimation of the operational sex ratios. On average there were seven males for each female present in the chorus. The males were apparently selected by females.

KEY WORDS. Hylidae, Scinax rizibilis, reproduction, sexual selection, spatial distribution, seasonality
\end{abstract}

Os machos de muitas espécies de anuros comumente formam agregações reprodutivas para a atração de fêmeas (BASTOS \& HADDAD 1996; WELLS 1977), sendo que os indivíduos podem apresentar algum grau de segregação espacial (DYSON \& PASSMORE 1992a) e temporal (AICHINGER 1987). A intensidade sonora das vocalizações parece ser o principal parâmetro utilizado pelos machos de anuros para avaliar a proximidade do vizinho (BRENOWITZ et al. 1984; GERHARDT et al. 1989). Além da vocalização, os combates físicos, em última instância, servem para manter a organização espacial (BASTOS \& HADDAD 1995; ROBERTSON 1986a).

Em muitas espécies de anuros, tanto com reprodução explosiva como prolongada (sensu WELLS 1977), a formação de casais pode não ocorrer de maneira aleatória (OLSON et al. 1986). Machos em amplexo podem ser maiores que os solitários (LEE \& CRUMP 1981) ou pode haver correlação entre os comprimentos ou massas de machos e de fêmeas em amplexo [escolha por classes de comprimento rostro-anal (CRA), ou por massa (LICHT 1976; ROBERTSON 1986b)]. Tanto a competição entre machos como a escolha realizada pelas fêmeas podem originar tal padrão não aleatório (OLSON et al. 1986).

A escolha realizada pela fêmea foi questionada como agente de seleção sexual (HALLIDAY 1983). Muitos estudos, entretanto, demonstraram a efícácia da

1) Departamento de Biologia Geral, Instituto de Ciências Biológicas, Universidade Federal de Goiás. Caixa Postal 131, 74001-970 Goiânia, Goiás, Brasil.

2) Departamento de Zoologia, Instituto de Biociências, Universidade Estadual Paulista. Caixa Postal 199, 13506-900 Rio Claro, São Paulo, Brasil. 
escolha realizada pela fêmea, fazendo com que haja, atualmente, um consenso de que as fêmeas, em muitas espécies, comparam as características dos machos, geralmente através de parâmetros acústicos, antes de se acasalarem (veja revisão em RYAN \& KEDDY-HECTOR 1992): Apesar de ter-se evidenciado, em experimentos de laboratórios, que fêmeas apresentam preferências por certas características dos cantos de machos, muitas vezes não se obtém os mesmos resultados em condições naturais (MORRIS \& YOON 1989). As agregações reprodutivas dos anuros podem, devido à sua complexidade, limitar a capacidade das fềmeas em distinguir os cantos dos machos (DYSON \& PASSMORE 1992b).

Neste trabalho examinouse a biologia reprodutiva de Scinax rizibilis (Bokermann, 1964), um hilídeo que ocorre na região de Ribeirão Branco, sul do estado de São Paulo. Os objetivos foram: (1) examinar a organização do coro em relação às distribuições espacial e temporal dos indivíduos; (2) determinar se os acasalamentos são aleatórios ou não em relação ao CRA ou à massa dos machos; (3) verificar se as fêmeas selecionam os machos através de escolha por classes de tamanho, em função do próprio CRA ou da massa destes.

\section{MATERIAL E MÉTODOS}

Realizamos os trabalhos de campo em uma poça temporária, com área aproximada de $1950 \mathrm{~m}^{2}$ e localizada na Fazendinha São Luís (24⒉ ' $\mathrm{S}, 48^{\circ} 44^{\prime} \mathrm{W}$; cerca de $800 \mathrm{~m}$ de altitude), $22 \mathrm{~km}$ distante da cidade de Ribeirão Branco, sul do Estado de São Paulo. O acesso à poça (a partir da sede) se fazia por uma trilha usada para extração de madeira. A poça era cercada por vegetação arbórea ou arbustiva, sendo que no seu interior, a distribuição da vegetação é, aparentemente, regular, com predominância da família Juncaceae.

A coleta regular de dados foi realizada entre fevereiro de 1993 e janeiro de $1994(\mathrm{n}=36$ turnos de observação, tabela I). Observações adicionais foram realizadas entre novembro de 1994 e fevereiro de 1995 ( $n=5$ turnos de observação). As visitas foram mensais na estação seca (entre março e agosto) e quinzenais na estação chuvosa (entre setembro e fevereiro) e consistiam de dois a seis turnos de observação, totalizando 148 horas (Tab. I).

Em três noites, ao longo do período de estudos, demarcamos uma área com $3 \mathrm{~m}^{2}$ na poça, dentro da qual todos os machos foram localizados e suas posições marcadas com uso de fita adesiva. Dessa forma, as distâncias para o vizinho mais próximo e a densidade de machos foram obtidas. Estes dados foram utilizados para a análise do padrão de distribuição espacial, onde $\mathrm{R}$ significa o grau com que a distribuição observada desvia-se da expectativa randômica (CLARK \& EvANS 1954). Também registramos as alturas em que os machos se encontravam.

Na maioria dos turnos de observação $(n=36)$, a poça foi sistematicamente percorrida para a coleta das seguintes informações: número de machos e de fêmeas, número de casais e deslocamentos efetuados pelos machos. Registramos a temperatura do ar (a $150 \mathrm{~cm}$ de altura) e da água (a no máximo $5 \mathrm{~cm}$ de profundidade), com termômetro de mercúrio com precisão de $0,5^{\circ} \mathrm{C}$. Para a realização das observações comportamentais, utilizamos os métodos de "animal focal", de "todas as ocorrências" e de "seqüências" (LEHNER 1979; MARTIN \& BATESON 1986). 
Tabela I. Turnos de observação, com respectivas horas de início e final, realizados durante o período de estudo, em Ribeirão Branco, São Paulo.

\begin{tabular}{|c|c|c|c|}
\hline Turno de observação & Mês & Inicio & Final \\
\hline 1 & Fevereiro & $17: 30$ & $22: 00$ \\
\hline 2 & Fevereiro & $17: 30$ & $22: 00$ \\
\hline 3 & Março & $17: 30$ & $20: 00$ \\
\hline 4 & Março & $17: 30$ & $20: 00$ \\
\hline 5 & Abril & $17: 40$ & $20: 10$ \\
\hline 6 & Abril & $18: 00$ & $20: 00$ \\
\hline 7 & Maio & $18: 00$ & $20: 00$ \\
\hline 8 & Maio & $18: 00$ & $19: 30$ \\
\hline 9 & Junho & $17: 50$ & $19: 20$ \\
\hline 10 & Junho & $18: 00$ & $20: 00$ \\
\hline 11 & Julho & $18: 00$ & $20: 00$ \\
\hline 12 & Julho & $18: 00$ & $20: 00$ \\
\hline 13 & Agosto & $18: 30$ & $20: 00$ \\
\hline 14 & Agosto & $18: 00$ & $20: 00$ \\
\hline 15 & Setembro & $19: 00$ & $23: 00$ \\
\hline 16 & Setembro & $18: 00$ & $24: 00$ \\
\hline 17 & Setembro & $18: 00$ & $24: 00$ \\
\hline 18 & Outubro & $18: 00$ & $03: 00$ \\
\hline 19 & Outubro & $19: 00$ & $03: 00$ \\
\hline 20 & Outubro & $18: 30$ & $01: 00$ \\
\hline 21 & Outubro & $18: 30$ & $02: 00$ \\
\hline 22 & Outubro & $19: 00$ & $24: 00$ \\
\hline 23 & Novembro & $18: 00$ & $20: 00$ \\
\hline 24 & Novembro & $18: 00$ & $20: 00$ \\
\hline 25 & Novembro & $18: 30$ & $20: 00$ \\
\hline 26 & Novembro & $18: 30$ & $20: 00$ \\
\hline 27 & Novembro & $18: 30$ & $20: 00$ \\
\hline 28 & Dezembro & $18: 00$ & $01: 00$ \\
\hline 29 & Dezembro & $19: 00$ & $03: 00$ \\
\hline 30 & Dezembro & $18: 00$ & $03: 30$ \\
\hline 31 & Dezembro & $19: 00$ & $03: 00$ \\
\hline 32 & Dezembro & $18: 00$ & $24: 00$ \\
\hline 33 & Dezembro & $18: 30$ & $22: 00$ \\
\hline 34 & Janeiro & $18: 00$ & $24: 00$ \\
\hline 35 & Janeiro & $18: 30$ & $23: 00$ \\
\hline 36 & Janeiro & $18: 00$ & $20: 00$ \\
\hline
\end{tabular}

Quando um casal em amplexo era encontrado, este era coletado, bem como os machos que se encontravam dentro de um raio de $30 \mathrm{~cm}$. A grande maioria desses indivíduos foi marcada por amputação de falanges (MARTOF 1953). O comprimento rostro-anal (CRA) foi medido com paquímetro (precisão de $0,1 \mathrm{~mm}$ ) e a massa com balança Pesola ${ }^{\circledR}$ (precisão de $0,05 \mathrm{~g}$ ), no dia subseqüente à captura. Para o cálculo aproximado da massa de ovos, pesou-se 16 fêmeas antes e após a oviposição. Todos os indivíduos foram soltos na noite consecutiva.

$\mathrm{O}$ índice de oportunidade de seleção sexual (Is) foi calculado (segundo ARNOLD \& WADE 1984) como sendo a variância no sucesso reprodutivo dos machos dividida pelo quadrado do sucesso reprodutivo médio dos machos. Como ao longo do estudo nem todos os machos foram marcados, para o cálculo do sucesso reprodutivo médio dos machos somente foram considerados os 25 machos em amplexo e os 59 machos solitários que foram coletados a $30 \mathrm{~cm}$ de distância do casal e cujas numerações eram reconhecidas. O sucesso reprodutivo médio dos machos foi obtido pela seguinte fórmula (WAGNER \& SULLIVAN 1992): 


$$
A=\frac{N A}{M A+M S} \times 100
$$

onde: (A) sucesso reprodutivo médio de todos os machos marcados; (NA) número de amplexos conseguidos pelos machos marcados; (MA) número de machos marcados que conseguiram amplexo; (MS) número de machos marcados que não conseguiram amplexo.

A razão sexual operacional (RSO) foi obtida pela divisão do número de fêmeas pelo número de machos presentes na agregação reprodutiva (EMLEN \& ORING 1977) para 16 noites.

Os dados são apresentados como média \pm desvio padrão (X $\pm \mathrm{DP})$. Para as análises estatísticas foram utilizadas as provas paramétricas do teste " $\mathrm{t}$ " e o coeficiente de correlação de Pearson (ZAR 1996) e a prova não paramétrica de KruskalWallis. O nível de significância considerado em todos os testes foi de $\mathrm{P}<0,05$.

\section{RESULTADOS}

\section{Características dos adultos}

As fêmeas de S. rizibilis eram maiores e mais pesadas, após a oviposição, do que os machos (Tab. II). Machos em amplexo eram mais pesados, porém não maiores, do que os machos solitários (Tab. III) ao longo de toda a estação. O valor médio da massa de ovos carregada pelas fêmeas foi de $0,64 \mathrm{~g}(\mathrm{n}=16)$, o que representa cerca de $50 \%$ da massa média das fêmeas após a oviposição.

O CRA foi positivamente correlacionado com a massa em machos solitários $(r=0,45 ; p<0,01 ; n=91)$, em machos em amplexo $(r=0,35 ; p<0,01 ; n=82)$ e em fêmeas $(r=0,53 ; p<0,01 ; n=68)$.

Tabela II. Comparação, pelo teste "t", dos valores médios de CRAs e massas de machos e fêmeas (após a oviposição) de Scinax rizibilis em Ribeirão Branco, São Paulo.

\begin{tabular}{|c|c|c|c|c|c|c|c|c|c|}
\hline & \multicolumn{3}{|c|}{ Machos } & \multicolumn{3}{|c|}{ Fêmeas } & \multirow{2}{*}{$t$} & \multirow{2}{*}{ gl } & \multirow{2}{*}{$P$} \\
\hline & $n$ & $\mathrm{X} \pm \mathrm{DP}$ & Amplitude & $\mathrm{n}$ & $X \pm D P$ & Amplitude & & & \\
\hline CRA (mm) & 189 & $26,65 \pm 0,94$ & $23,90-29,50$ & 82 & $32,51 \pm 1,13$ & $29,50-35,80$ & 41,05 & 269 & $<0,01$ \\
\hline Massa $(g)$ & 188 & $0,87 \pm 0,12$ & $0,45-1,55$ & 68 & $1,39 \pm 0,19$ & $0,90-1,85$ & 25,79 & 254 & $<0,01$ \\
\hline
\end{tabular}

Tabela III. Comparação, pelo teste "t", dos valores médios de CRAs e massas de machos em amplexo e de machos solitários de Scinax rizibilis em Ribeirão Branco, São Paulo.

\begin{tabular}{|c|c|c|c|c|c|c|c|c|c|}
\hline & \multicolumn{3}{|c|}{ Machos } & \multicolumn{3}{|c|}{ Fêmeas } & \multirow{2}{*}{$t$} & \multirow{2}{*}{$\mathrm{gl}$} & \multirow{2}{*}{$P$} \\
\hline & $n$ & $\mathrm{X} \pm \mathrm{DP}$ & Amplitude & $n$ & $X \pm D P$ & Amplitude & & & \\
\hline $\mathrm{CRA}(\mathrm{mm})$ & 83 & $26,54 \pm 0,97$ & $23,90-29,50$ & 106 & $26,70 \pm 1,06$ & $21,20-29,21$ & 1,08 & 82 & $>0,05$ \\
\hline Massa $(\mathrm{g})$ & 82 & $0,89 \pm 0,13$ & $0,65-1,55$ & 106 & $0,84 \pm 0,12$ & $0,45-1,05$ & 2,53 & 160 & $<0,05$ \\
\hline
\end{tabular}

\section{Estrutura do coro}

Indivíduos de $S$. rizibilis apresentaram atividade reprodutiva (indicada pela presença de machos vocalizantes e de fềmeas com ovos) nos meses quentes e chuvosos do ano (Figs 1-2). Entre os meses de março e agosto de 1993 não houve 


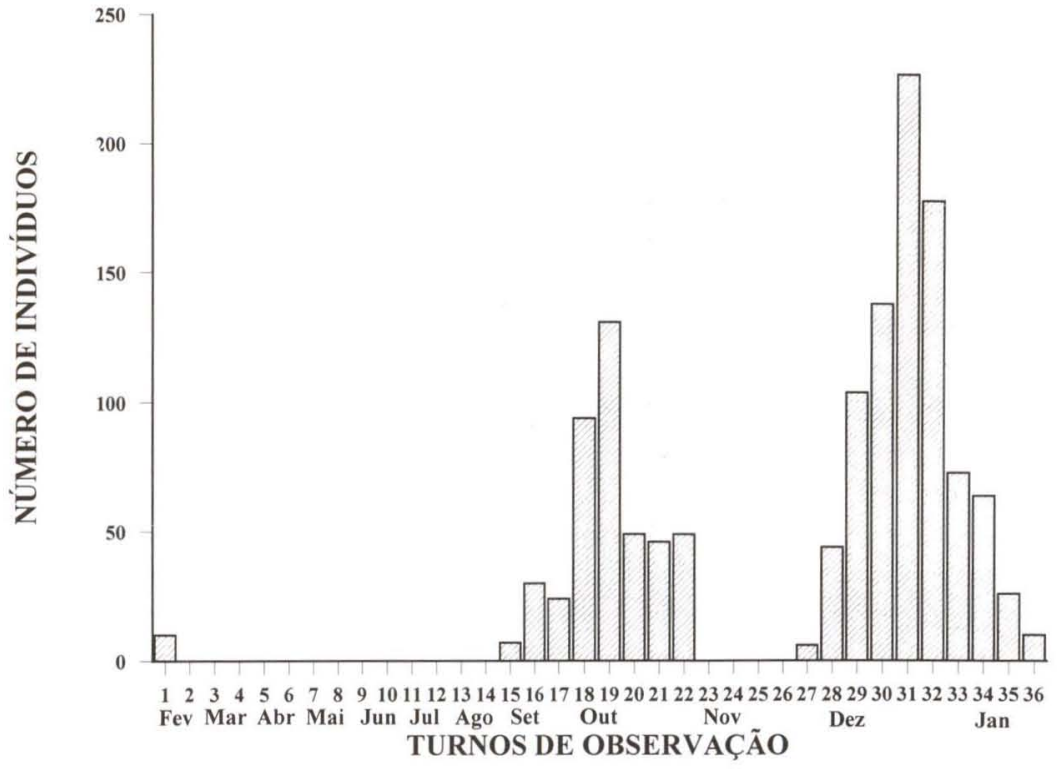

Fig. 1. Número de individuos de Scinax rizibilis observados no local, em cada turno, entre fevereiro de 1993 e janeiro de 1994 em Ribeirão Branco, São Paulo.

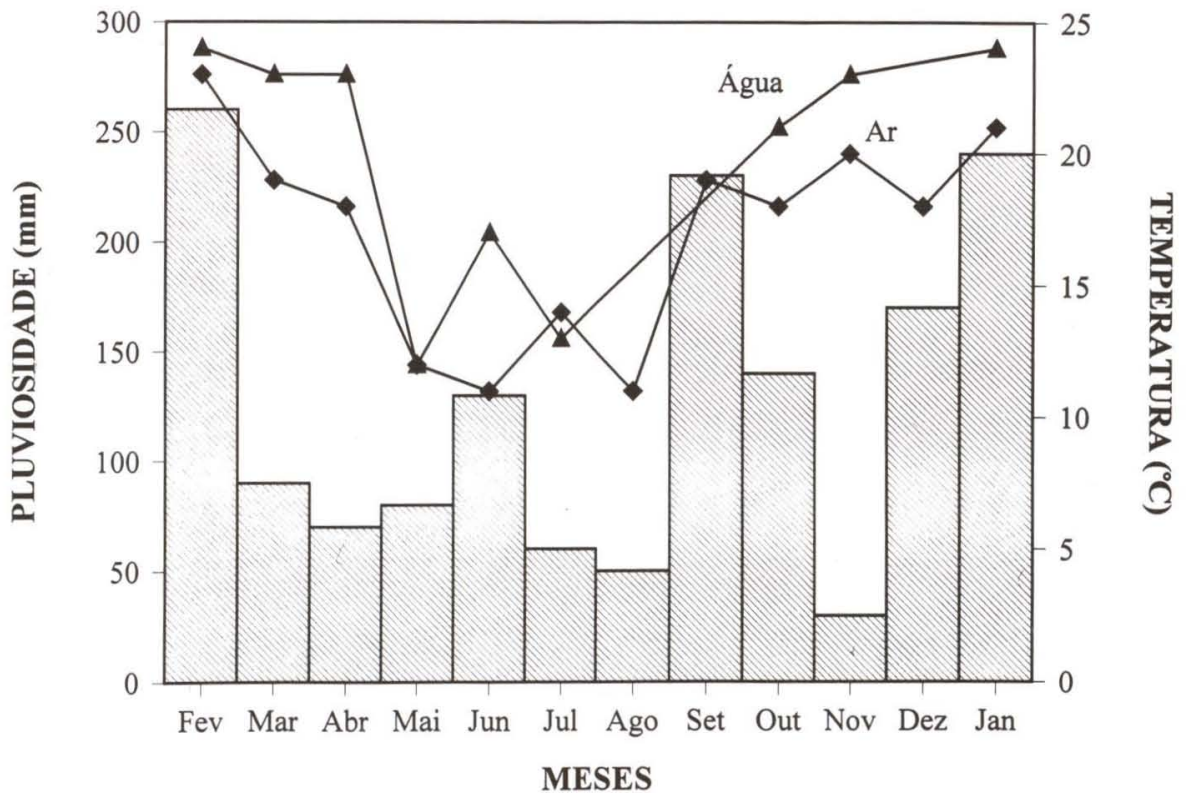

Fig. 2. Pluviosidade (barras), temperatura do ar e da água, registradas entre fevereiro de 1993 e janeiro de 1994, em Ribeirão Branco, São Paulo. 
nenhuma atividade da espécie na poça, que esteve seca entre julho e agosto. Os meses entre maio e agosto foram os mais frios e os de julho, agosto e novembro com menor pluviosidade (Fig. 2). Os primeiros machos foram observados em vocalização a partir de setembro. Neste mês também foram observadas as primeiras fềmeas com óvulos maduros, mas nenhum casal em amplexo foi encontrado.

No coro, os machos ocupavam, como sítios de vocalização, ramos da vegetação arbustiva marginal ou emergente, localizados a uma altura de 10 a 100 $\mathrm{cm}$ do solo ou da água $(46 \pm 23 \mathrm{~cm} ; \mathrm{n}=60)$ e agrupavam-se preferencialmente em determinadas áreas. Estes agrupamentos não eram fixos, havendo ocupações de diferentes áreas no decorrer da estação reprodutiva.

A distância entre os machos variou de 8 a $58 \mathrm{~cm}(28 \pm 10 \mathrm{~cm} ; n=62)$, sendo raro encontrar dois machos a menos de $10 \mathrm{~cm}$ um do outro. A distribuição espacial dos machos tendia a ser uniforme (valores de $\mathrm{R}$ variando de 1,42 a 1,52, tabela IV), mesmo nos coros com maior densidade $\left(8,33\right.$ machos $\left./ \mathrm{m}^{2}\right)$. Apesar de ter havido aumento na densidade de machos, não ocorreu diferença significativa entre as distâncias interindividuais médias registradas $(\mathrm{H}=2,05 ; \mathrm{p}>0,05$; Kruskal-Wallis).

Tabela IV. Resultados das análises sobre distância de vizinhos mais próximos de Scinax rizibilis, em três noites, em Ribeirão Branco, São Paulo.

\begin{tabular}{|c|c|c|c|c|c|c|}
\hline \multirow{2}{*}{ Data } & \multirow{2}{*}{$\begin{array}{c}\text { Tamanho da amostra } \\
\text { observada do coro }\end{array}$} & \multirow{2}{*}{$\frac{\text { Diståncia entre os machos }(\mathrm{cm})}{X_{ \pm} \mathrm{DP}}$} & \multirow{2}{*}{$\begin{array}{c}\text { Densidade } \\
\left(\mathrm{m}^{2}\right)\end{array}$} & \multirow{2}{*}{$\mathrm{R}^{(1)}$} & \multirow{2}{*}{$c^{(2)}$} & \multirow{2}{*}{ Interpretaçăo } \\
\hline & & & & & & \\
\hline $09 / \times / 93$ & 22 & $31,87 \pm 13,68$ & 5,00 & 1,42 & 3,15 & Uniforme \\
\hline $30 / x / 93$ & 32 & $27,04 \pm 9,35$ & 7,33 & 1,46 & 4,17 & Uniforme \\
\hline $25 / X 11 / 93$ & 55 & $26,88 \pm 12,30$ & 8,33 & 1,59 & 6,54 & Uniforme \\
\hline
\end{tabular}

(1) Segundo ClaRK \& Evans (1954); (2) valores críticos para "c" de desvio da curva normal são: $1,96, p<0,05$ e $2,58, p<0,01$

O número de machos presente no coro foi positivamente correlacionado com o número de fêmeas encontrado $(\mathrm{r}=0,92 ; \mathrm{p}<0,01 ; \mathrm{n}=16)$. Em $84 \%$ das noites em que houve atividade de vocalização, as fêmeas estavam presentes no coro. Nessas agregações, havia sempre maior número de machos de que de fêmeas (em média 7 machos: 1 fêmea), o que resultou em razões sexuais operacionais (RSOs) muito baixas. A correlação entre RSO e número de machos não foi significativa $(r=0,28$; $\mathrm{p}>0,05 ; \mathrm{n}=16)$, sugerindo que não houve, proporcionalmente, mais fêmeas em coros grandes.

\section{Comportamento de machos e fêmeas no coro e sistema de acasalamento}

Os machos de $S$. rizibilis vocalizavam, geralmente, a partir da vegetação marginal arbustiva ou emergente. Alguns machos permaneciam imóveis por até seis horas nos ramos de vegetação ou realizavam deslocamentos pela área ocupada pelo coro. Como não observamos o início da formação de casais, não é possível afirmar se as fêmeas com ovos iniciavam o amplexo ou se os machos as interceptavam. Algumas dessas fêmeas $(n=3)$ foram observadas se deslocando pela área do coro sem serem interceptadas, outras $(n=5)$ permaneciam imóveis por até quatro horas. Em uma ocasião, um macho foi observado sobre um casal em amplexo, não sendo possível afirmar se estava tentando deslocar o macho em amplexo. 
No laboratório, entretanto, um macho foi observado tentando deslocar o macho de um casal em amplexo. O macho "deslocador" tentava colocar a cabeça e os braços sob o ventre do macho em amplexo, que desferia golpes de pernas no deslocador. Nesta observação, o macho deslocador, o qual não foi bem sucedido, apresentava valores de CRA e de massa $(26,3 \mathrm{~mm}$ e $0,90 \mathrm{~g})$ similares aos do macho em amplexo (26,8 mm e $0,88 \mathrm{~g})$.

Oitenta e dois casais foram capturados em amplexo. Durante o amplexo, o macho segura firmemente as axilas da fêmea com as mãos, sendo que os seus polegares apresentam calosidades nupciais, que poderiam propiciar uma maior aderência à pele da fềmea. Somente um macho foi observado duas vezes em amplexo. A média de sucesso reprodutivo dos machos foi de $30 \%$, produzindo um valor de oportunidade de seleção sexual (Is) de 2,36.

Considerando toda a estação reprodutiva, o padrão de acasalamentos observado para $S$. rizibilis não foi aleatório. Machos em amplexo foram mais pesados que os machos solitários; entretanto, não houve diferença significativa entre os CRAs (Tab. III). Também houve correlação positiva entre as massas de machos e de fêmeas em amplexo $(r=0,46 ; n=66 ; p<0,05)$ e entre os CRAs $(r=0,24 ; n=$ $81 ; \mathrm{p}<0,05)$, caracterizando escolha por classes de machos ("assortative mating" sensu PARTRIDGE 1983).

\section{DISCUSSÃo}

\section{Estrutura do coro}

As agregações reprodutivas de $S$. rizibilis podem ser consideradas como "leks" (sensu EMLEN \& ORING 1977; BRAdBuRY 1981; Sullivan \& Hinshaw 1992), pois os sítios de vocalização dos machos podem ser considerados como áreas de exibição. Dentro dessas agregações, as distribuições dos machos tendiam a ser uniformes. Pelo método de CLARK \& EvANS (1954), um valor de R ao redor de 1 indica distribuição aleatória e a distribuição uniforme produz resultado igual a 2,14 (uniformidade máxima). Valores intermediários entre 1 e 2 indicam distribuição tendendo à uniformidade. O padrão de distribuição apresentado por machos de $S$. rizibilis tendia à uniformidade, indicando que os indivíduos são capazes de manter uma separação espacial padronizada entre eles. A manutenção desse espaçamento poderia limitar o número de participantes e, conseqüentemente, o número de competidores no coro (DYSON \& PASSMORE 1992a; WHITNEY \& KREBS 1975), bem como possibilitar que cada macho fosse acusticamente distinguível de seus vizinhos, para as fêmeas (DYSON \& PASSMORE 1992b; ROBERTSON 1984).

Machos de diversas espécies de Hylidae vocalizam a partir de sítios elevados (GREER \& WELLS 1980; MitCHELl 1991; NARINS \& HURLEY 1982), talvez porque o som seja melhor propagado nestas condições (WELLS \& SCHWARTZ 1982), pois ocorre grande atenuação dos sons produzidos próximos ao substrato (MITCHELL 1991). Dessa forma, a altura de um sítio de canto poderia ser tão importante para um macho de S. rizibilis quanto o tipo de vegetação, pois influenciando na distância em que ocorreria a propagação do som, poderia interferir na parte final de atração de fêmeas, como ocorre em outras espécies (GREER \& WELLS 1980; ROBERTSON 1984). 
Quanto ao padrão temporal de reprodução, os anuros podem apresentar duas estratégias: a de reprodução prolongada e a de reprodução explosiva (WELLS 1977). De uma maneira geral, nas espécies com estação reprodutiva explosiva há intensa competição entre machos, chegada sincrônica de machos e de fềmeas no sítio reprodutivo, razões sexuais operacionais altas e baixas oportunidades para seleção sexual por escolha realizada pelas fêmeas (ARAK 1983; OLsON et al. 1986). As espécies com estação reprodutiva prolongada, por sua vez, estão associadas com chegada assincrônica de machos e de fêmeas, escolha de machos realizada pelas fêmeas, razões sexuais operacionais baixas e maior oportunidade de seleção sexual por escolha realizada pelas fêmeas (ARAK 1983; WELLS 1977).

Embora essa classificação ainda seja útil, diversos estudos demonstraram que há dificuldades em estabelecer o padrão de uma espécie e que também há grande variação nesse padrão entre as populações (HOWARD 1988; WOODWARD 1982). A população estudada de $S$. rizibilis apresenta tanto características de reprodução prolongada (reprodução por várias semanas, defesa de território e RSOs baixas) como de reprodução explosiva (sincronismo elevado na chegada de machos e de fêmeas e atividade reprodutiva ao longo do dia e da noite).

As razões sexuais operacionais foram pequenas, havendo grande número de machos em relação ao de fêmeas, como ocorre em outras espécies de reprodução prolongada (DYSON et al. 1992; POMBAL et al. 1994). Em média, nas agregações reprodutivas de S. rizibilis, havia sete vezes mais machos que fêmeas. No entanto, houve correlação positiva entre o número de machos e o número de fêmeas presentes no coro, de maneira similar ao observado para outras espécies (BASTOS \& HADDAD 1996; Sullivan \& Hinshaw 1992; TEJEDO 1993). Tal padrão pode ser devido, a pelo menos duas razões: (1) fêmeas preferem participar de agregações maiores, pois nestas os acasalamentos são mais fáceis de serem conseguidos (BRADBURY 1981) e (2) coros maiores são mais audiveis e, assim, atraem mais fêmeas (WELLS 1977).

A chegada das fêmeas de $S$. rizibilis ao sítio reprodutivo foi bastante sincrônica com a presença dos machos; as fêmeas estavam presentes em $84 \%$ das noites em que houve atividade do coro (dois, ou mais, machos vocalizando). Este resultado é maior do que o observado em outros hilídeos neotropicais, como Hyla elegans (58\%, BASTOS \& HADDAD 1995) e H. faber (42\%, MARTINS 1993). O maior sincronismo na chegada de machos e fêmeas ao local de reprodução, propicia maior número de desovas simultâneas no ambiente, o que poderia reduzir a predação de ovos e de girinos (WELLS 1979), devido a saciação dos predadores.

O sistema reprodutivo apresentado por uma espécie de anuro pode influenciar a oportunidade de seleção sexual (WAGNER \& SULLIVAN 1992). Esta influência sobre o Is pode ser decorrente da RSO, do grau de sincronismo da chegada de machos e de fêmeas no coro e da duração da estação reprodutiva. Baixas estimativas de RSO, indicam que a média de sucesso reprodutivo dos machos é pequena, resultando em Is elevado. Além disso, em espécies com reprodução prolongada, a chegada das fêmeas torna-se assincrônica à medida que a estação reprodutiva se estende, fazendo com que o Is aumente porque alguns machos podem se reproduzir mais de uma vez. Dessa forma, teoricamente, o Is poderia fornecer indicações do padrão reprodutivo temporal apresentado por uma população de anuro, isto é: espécies com Is baixos 
seriam de reprodução explosiva e espécies com Is elevado seriam de reprodução prolongada.

Todavia, ao se comparar Is de diversas espécies de anuros (Tab. V) verifica-se que não é possível estabelecer um padrão para espécies de reprodução prolongada ou explosiva. Veja que os valores de Is para espécies com reprodução explosiva ou prolongada podem ser similares. Esta similaridade talvez seja decorrente das diferentes metodologias utilizadas nos diferentes trabalhos. Poucos são os trabalhos que se extendem por mais de uma estação reprodutiva ou mais de um sítio reprodutivo e, em muitos dos trabalhos realizados no Brasil (inclusive este), as visitas ao local de estudo foram realizadas com grande intervalo de tempo.

Tabela V. Comparação de valores do indice de oportunidade de seleção sexual (Is) para diferentes espécies de anuros

\begin{tabular}{llcl}
\hline \multicolumn{1}{c}{ Espécie } & Padrão reprodutivo & Is & Referéncia \\
\hline $\begin{array}{l}\text { Bufonidae } \\
\text { Bufo americanus }\end{array}$ & Explosivo & 5,87 & GATZ (1981) \\
$\begin{array}{l}\text { Bufo bufo } \\
\text { Bufo valliceps }\end{array}$ & Explosivo & 4,78 & DAVIES \& HALLIDAY (1979) \\
Hylidae & Explosivo (?) & $2,28-32,22$ & WAGNER \& SULLIVAN (1992) \\
$\begin{array}{l}\text { Hyla elegans } \\
\text { H. faber }\end{array}$ & Prolongado & 5,74 & BASTOS \& HADDAD (1996) \\
$\begin{array}{l}\text { Scinax rizibilis } \\
\text { Leptodactylidae }\end{array}$ & Prolongado & 19,20 & MARTINS (1993) \\
$\begin{array}{l}\text { Physalaemus pustulosus } \\
\text { Ranidae }\end{array}$ & Intermediário & 2,36 & Presente estudo \\
Rana catesbeiana & Prolongado & 17,51 & RYAN (1983) \\
\hline
\end{tabular}

\section{Comportamento do macho e da fêmea no coro e sistema de acasalamento}

Em muitas espécies de anuros, os acasalamentos ocorrem de maneira não aleatória (HowARD \& KLUGE 1985; OLSON et al. 1986), podendo ser evidenciada vantagem reprodutiva dos machos0 maiores e/ou acasalamentos por classes de CRA ou massa ("assortative mating" sensu PARTRIDGE 1983). Os dois mecanismos de seleção sexual (competição entre machos e escolha realizada pela fềmea) podem estar envolvidos (DAVIES \& HALLIDAY 1977; LEE \& CRUMP 1981).

A despeito de não ter sido observado o início do amplexo em S. rizibilis, algumas evidências indicam a participação desses dois mecanismos. Para a competição entre machos, as evidências foram: (1) estimativas baixas de RSO (WELLS 1977), (2) tentativa de desalojamento do macho amplectante observada em laboratório (DAVIES \& HALLIDAY 1979); (3) defesa de territórios através de vocalizações ou comportamentos agressivos (competição por interferência BEGON et al. 1996). Para a escolha realizada pela fêmea, a única indicação foi a de que as fêmeas podiam se deslocar entre os territórios dos machos sem que fossem interceptadas (HowARD 1988).

Algumas outras evidências, tanto para a competição entre machos como para a escolha realizada pela fềmea, têm sido propostas na literatura; todavia, estas podem não determinar que um ou outro componente esteja atuando. Por exemplo, a competição entre machos tem sido evidenciada pela comparação de valores médios do CRA e da massa de machos em amplexo e de machos solitários, sendo os maiores 
e os mais pesados, com maior sucesso reprodutivo (ARAK 1983). No entanto, deve-se considerar que a escolha realizada pela fêmea também poderia resultar em tal padrão não aletório (RYAN 1983).

Para S. rizibilis tanto as massas como os CRAs de machos e de fềmeas em amplexo foram significativamente correlacionadas ao longo da estação. Em muitas espécies de anuros, a freqüência dominante dos cantos é correlacionada negativamente com o CRA dos machos (RYAN 1985; SULLIVAN \& WAGNER 1988; WAGNER 1989) ou com a massa (GIVEN 1987). Se as fêmeas de S. rizibilis são capazes de avaliar os machos utilizando a freqüência dominante das suas vocalizações e, se esta é correlacionada com o CRA ou massa dos machos, o resultado obtido seria esperado (correlação entre as massas como também entre CRAs de machos e de fêmeas em amplexo).

O fato da correlação entre as massas de machos e de fêmeas em amplexo ter sido maior que a correlação entre os CRAs, pode estar relacionado às baixas correlações observadas entre CRA e massa dos machos em amplexo e dos machos solitários. Essas últimas correlações indicam que há uma grande variação da massa em relação ao CRA, pois um macho que conseguiu amplexo, poderia ter perdido peso durante a sua presença no coro, na tentativa de manter um território. Dessa forma, machos muito grandes podem apresentar pesos muito pequenos. Como o CRA de algumas espécies de anuros, praticamente permanece invariável ao longo de uma estação reprodutiva (ROBERTSON 1986a), as fêmeas de $S$. rizibilis tenderiam avaliar os machos mais realisticamente pela diferença de suas massas, uma vez que machos maiores teriam cordas vocais de maior massa e, conseqüentemente, cantos de freqüência menor (MARTIN 1972).

A variabilidade no padrão de acasalamento, ao longo da estação reprodutiva, observada para S. rizibilis é comum entre os anuros (OLSON et al. 1986). Diversos fatores podem estar envolvidos na causa dessa variabilidade, além da seleção sexual. Por exemplo, o padrão de acasalamento pode estar relacionado com a extensão da estação reprodutiva (LEE \& CRUMP 1981). A maioria das espécies com variabilidade no padrão de acasalamento apresenta reprodução explosiva, indicando que estas espécies são mais propensas a alterarem o padrão devido a uma maior sensibilidade às flutuações das condições ambientais ou devido a processos dependentes de densidade (OLSON et al. 1986).

AGRADECIMENTOS. Agradecemos a J.A.F. Diniz-Filho, J.P. Pombal Jr. e L.M. Bini pela leitura e sugestões apresentadas ao manuscrito. A J.P. Pombal Jr., E.C.P. Pombal, O.C. Oliveira, F. Baldissera pela ajuda nos trabalhos de campo. A A.T.d'Heursel pela ajuda na confecção do "abstract". Aos Srs Newton e Nilson Mattedi pela permissão de acesso ao local de estudos. A FAPESP (proc. 92/0631-2, 94/5634-5 e 95/9269-2) pelo suporte financeiro. Ao CNPq e CAPES (PICD) pelas bolsas concedidas.

\section{REFERÊNCIAS BIBLIOGRÁFICAS}

AICHINGER, M. 1987. Annual activity patterns of anurans in a seasonal Neotropical environment. Oecologia 71: 583-592.

Revta bras. Zool. 16 (2): 409 - 421, 1999 
ARAK, A. 1983. Male-male competition and mate choice in anuran amphibians, p.181-210. In: P. BATESON (Ed.). Mate choice. Cambridge, Cambridge Univ. Press, 462p.

ARNOLD, S.J. \& M.J. WADE. 1984. On the measurement of natural and sexual selection: theory. Evolution 38: 709-719.

BASTOS, R.P. \& C.F.B. HADDAD. 1995. Vocalizações e interações acústicas em Hyla elegans (Anura, Hylidae) durante a atividade reprodutiva. Naturalia 20: 165-176.

. 1996. Breeding activity of the neotropical treefrog Hyla elegans (Anura, Hylidae). Jour. Herpetol. 30 (3): 355-360.

BEgON, M.; J.L. HARPER \& C.R. TownSEND. 1996. Ecology. Oxford, Blackwell Science, 1068p.

BRADBURY, J.W. 1981. The evolution of leks, p.138-169. In: R.D. ALEXANDER \& D.W. TINKLE (Eds). Natural selection and social behavior: recent reasearch and new theory. New York, Chiron Press, 340p..

BRENOWITZ, E.A.; W. WILCZYNSKI \& H.H. ZAKON. 1984. Acoustic communication in spring peepers: environmental and behavioral aspects. Jour. Comp. Physiol. A 155: 585-592.

Clark, P.J. \& F.C. Evans. 1954. Distance to nearest neighbor as a measure of spatial relationships in populations. Ecology 35 (4): 445-453.

DAVIES, N.B. \& T.R. HALLIDAY. 1977. Optimal mate selection in the toad Bufo bufo. Nature 269: 56-58.

1979. Competitive mate searching in male common toads, Bufo bufo. Anim. Behav. 27: 1253-1267.

DYSON, M.L. \& N.I. PASSMORE. 1992a. Inter-male spacing and aggression in African painted reed frogs, Hyperolius marmoratus. Ethology 91: 237-247.

- 1992b. Effect of intermale spacing on female frequency preferences in the painted reed frog. Copeia 1992 (4): 1111-1114.

DysOn, M.L.; N.I. PASSMORE; P.J. BISHOP \& S.P. HENZI. 1992. Male behavior and correlates of mating success in a natural population of African painted reed frogs (Hyperolius marmoratus). Herpetologica 48 (2): 236-246.

EMLEN, S.T. \& L.W. ORING. 1977. Ecology, sexual selection and the evolution of mating systems. Science 197: 215-223.

GATZ JR., A.J. 1981. Non-randon mating by size in American toads, Bufo americanus. Anim. Behav. 29: 1004-1012.

GERHARDT, H.C.; B. DIEKAMP \& M. PTACEK. 1989. Inter-male spacing in choruses of the spring peeper, Pseudacris (Hyla) crucifer. Anim. Behav. 38: 1012-1024.

GIVEN, M.F. 1987. Vocalizations and acoustic interactions of the carpenter frog, Rana virgatipes. Herpetologica 43 (4): 467-481.

GREER, B.J. \& K.D. WELLS. 1980. Territorial and reproductive behavior of the tropical American frog Centrolenella fleischmanni. Herpetologica 36 (4): 318-326.

HallidAY, T.R. 1983. Do frogs and toads choose their mates? Nature 306: 226-227.

HowARD, R.D. 1979. The evolution of mating strategies in bullfrogs, Rana catesbeiana. Evolution 32 (4): 850-871. 
1988. Sexual selection on male body size and mating behaviour in American toads, Bufo americanus. Anim. Behav. (36): 1796-1808.

HowARD, R.D. \& A.G. KLUGE. 1985. Proximate mechanisms of selection in wood frogs. Evolution 39 (2): 260-277.

LEE, J.C. \& M.L. CRUMP. 1981. Morphological correlates of male mating success in Triprion petasatus and Hyla marmorata (Anura: Hylidae). Oecologia 50: 153-157.

LEHNER, P.N. 1979. Handbook of ethological methods. New York, Garland STPM Press, 403p.

LiCHT, L.E. 1976 Sexual selection in toads (Bufo americanus). Can. Jour. Zool. 54: $1277-1284$.

MARTIN, W.F. 1972. Evolution of vocalizations in the genus Bufo, 279-309p. In: W.F. BLAIR (Ed.) Evolution in the genus Bufo. Austin, University of Texas Press, 460p.

MARTIN, P. \& P. BATESON. 1986 Measuring behaviour. An introdutory guide. Cambridge, Cambridge Univ. Pres, 199p.

MARTINS, M. 1993. Observations on the reproductive behaviour of the Smith frog, Hyla faber. Herpetol. Jour. 3: 31-34.

MARTOF, B.S. 1953. Territoriality in the green frog, Rana clamitans. Ecology 34: 165-174.

MitCHELL, S.L. 1991. Intermale spacing and calling site characteristics in a Southern Mississipi chorus of Hyla cinerea. Copeia 1991 (2): 521-524.

MORRIS, M.R. \& S.L. YOON. 1989. A mechanism for female choice of large males in the treefrog Hyla chrysoscelis. Behav. Ecol. Sociobiol. 25: 502-519.

NARINS, P.M. \& D.D. HURLEY. 1982. The relationship between call intensity and function in the Puerto Rican coqui (Anura: Leptodactylidae). Herpetologica 38: 287-295.

OLsON, D.H.; A.R. BlaUsteIn \& R.K. O'HARA. 1986. Mating pattern variability among western toad (Bufo boreas) populations. Oecologia 70: 351-356.

PARTRIDGE, L. 1983. Non-random mating and offspring fitness, p.227-256. In:: P. Bateson (Ed.). Mate choice. Cambridge, Cambridge Univ. Press, 462p.

PomBal JR., J.P.; I. SAZIMA \& C.F.B. HADDAD. 1994. Breeding behavior of the pumpkin toadlet, Brachycephalus ephippium (Brachycephalidae). Jour. Herpetol. 28 (4): 516-519.

ROBERTSON, J.G.M. 1984. Acoustic spacing by breeding males of Uperoleia rugosa (Anura: Leptodactylidae). Z. Tierpsychol. 64: 283-297.

. 1986a. Male territoriality, fighting and assessment of fighting ability in the Australian frog Uperoleia rugosa. Anim. Behav. 39: 639-645.

. 1986b. Female choice, male strategies and the role vocalizations in the Australian frog Uperoleia rugosa. Anim. Behav. 34: 773-784.

RYAN, M.J. 1983. Sexual selection and communication in a Neotropical frog, Physalaemus pustulosus. Evolution 37 (2): 261-272.

- 1985. The tungara frog. a study in sexual selection and communication. Chicago, University of Chicago Press, 230p.

RYAN, M.J. \& A. KEDDY-HECTOR. 1992. Directional patterns of female mate choice and the role of sensory biases. Amer. Nat. 139 (Supl.): 4-35. 
SULLIVAN, B.K. \& W.E. WAGNER JR. 1988. Variation in advertisement and release calls, and social influences on calling behavior in the gulf coast toad (Bufo valliceps). Copeia 1988: 1016-1022.

Sullivan, B.K. \& S.H. Hinshaw. 1992. Female choice and selection on male calling behaviour in the gray treefrog Hyla versicolor. Anim. Behav. 44: 733-744.

TeJEDO, M. 1993. Do male Natterjack toads join larger breeding choruses to increase mating success? Copeia 1993 (1): 75-80.

WAGNER JR., W.E. 1989. Fighting, assessment, and frequency alteration in blanchard's cricket frog. Behav. Ecol. Sociobiol. 25: 429-436.

WAGNER JR., W.E. \& B.K. SUlLIVAN. 1992. Chorus organization in the gulf coast toad (Bufo valliceps): male and female behavior and the opprtunity for sexual selection. Copeia 1992 (3): 647-658.

WELLS, K.D. 1977. The social behaviour of anuran amphibians. Anim. Behav. 25: 666-693.

-1979. Reproductive behavior and male mating success in a Neotropical toad Bufo typhonius. Biotropica 11 (4): 301-307.

WELLS, K.D. \& J.J. SCHWARTZ. 1982. The effect of vegetation on the propagation of calls in the Neotropical frog Centrolenella fleischmanni. Herpetologica 38 (4): 449-455.

WhitNeY, C.L. \& J.R. KREBS. 1975. Spacing and calling in Pacific tree frogs, Hyla regilla. Can. Jour. Zool. 53: 1519-1527.

WOODWARD, B.D. 1982. Sexual selection and nonrandom mating patterns in desert anurans (Bufo woodhousei, Scaphiopus couchi and S. bombifrons). Copeia 1982: 351-355.

ZAR, J.H. 1996. Biostatistical Analysis. Englewood Cliffs, Prentice-Hall, 818p.

Recebido em 17.X.1997; aceito em 25.III.1999. 